\title{
Two explicit formulas for the generalized Motzkin numbers
}

\author{
Jiao-Lian Zhao ${ }^{1,2^{*}}$ and Feng Qij ${ }^{3,4}$ [
}

\section{"Correspondence:}

zhaoj|2004@gmail.com

1 State Key Laboratory of Integrated Service Networks, Xidian University,

Xi'an, Shaanxi 710071, China

${ }^{2}$ Department of Mathematics and

Physics, Weinan Normal University,

Weinan, Shaanxi 714009, China

Full list of author information is

available at the end of the article

\begin{abstract}
In the paper, by the Faà di Bruno formula, the authors establish two explicit formulas for the Motzkin numbers, the generalized Motzkin numbers, and the restricted hexagonal numbers.

MSC: Primary 05A15; secondary 05A19; 05A20; 05A15; 11B37; 11B83

Keywords: explicit formula; Motzkin number; generalized Motzkin number; restricted hexagonal number; Catalan number; generating function; Faà di Bruno formula; Bell polynomial of the second kind
\end{abstract}

\section{Introduction and main results}

The Motzkin numbers $M_{n}$ enumerate various combinatorial objects. In 1977, fourteen different manifestations of the Motzkin numbers $M_{n}$ were given in [1]. In particular, the Motzkin numbers $M_{n}$ give the numbers of paths from $(0,0)$ to $(n, 0)$ which never dip below the $x$-axis $y=0$ and are made up only of the steps $(1,0),(1,1)$, and $(1,-1)$.

The first seven Motzkin numbers $M_{n}$ for $0 \leq n \leq 6$ are 1,1,2, 4, 9, 21, 51. All the Motzkin numbers $M_{n}$ can be generated by

$$
M(x)=\frac{1-x-\sqrt{1-2 x-3 x^{2}}}{2 x^{2}}=\frac{1}{1-x+\sqrt{1-2 x-3 x^{2}}}=\sum_{k=0}^{\infty} M_{k} x^{k} .
$$

They can be connected with the Catalan numbers

$$
C_{n}=\frac{1}{n+1}\left(\begin{array}{c}
2 n \\
n
\end{array}\right)
$$

by

$$
M_{n}=\sum_{k=0}^{\lfloor n / 2\rfloor}\left(\begin{array}{c}
n \\
2 k
\end{array}\right) C_{k} \quad \text { and } \quad C_{n+1}=\sum_{k=0}^{n}\left(\begin{array}{l}
n \\
k
\end{array}\right) M_{k},
$$

where $\lfloor x\rfloor$ denotes the floor function whose value is the largest integer less than or equal to $x$. For detailed information, please refer to [2] and the closely related references therein. For information on many results, applications, and generalizations of the Catalan numbers $C_{n}$, please refer to the monographs [3, 4], the papers [5-13], the survey article [14], and the closely related references therein.

(c) The Author(s) 2017. This article is distributed under the terms of the Creative Commons Attribution 4.0 International License (http://creativecommons.org/licenses/by/4.0/), which permits unrestricted use, distribution, and reproduction in any medium, provided you give appropriate credit to the original author(s) and the source, provide a link to the Creative Commons license, and indicate if changes were made. 
In [15], the $(u, l, d)$-Motzkin numbers $m_{n}^{(u, l, d)}$ were introduced and it was shown in [15], Theorem 2.1, that $m_{n}^{(u, l, d)}=m_{n}^{(1, l, u d)}$,

$$
M_{u, l, d}(x)=\frac{1-l x-\sqrt{(1-l x)^{2}-4 u d x^{2}}}{2 u d x^{2}}=\sum_{n=0}^{\infty} m_{n}^{(u, l, d)} x^{n}
$$

and

$$
m_{n}^{(u, l, d)}=l^{n} \sum_{j=0}^{n / 2} \frac{1}{j+1}\left(\begin{array}{c}
2 j \\
j
\end{array}\right)\left(\begin{array}{l}
n \\
2 j
\end{array}\right)\left(\frac{u d}{l^{2}}\right)^{j}
$$

Comparing (1.1) with (1.2) reveals that $m_{n}^{(1,1,1)}=M_{n}$ and the $(u, l, d)$-Motzkin numbers $m_{n}^{(u, l, d)}$ generalize the Motzkin numbers $M_{n}$.

In [16], the Motzkin numbers $M_{n}$ were generalized in terms of the Catalan numbers $C_{n}$ to

$$
M_{n}(a, b)=a^{n} \sum_{k=0}^{\lfloor n / 2\rfloor}\left(\begin{array}{c}
n \\
2 k
\end{array}\right)\left(\frac{b}{a^{2}}\right)^{k} C_{k}
$$

for $a, b \in \mathbb{N}$ and the generating function

$$
M_{a, b}(x)=\frac{1-a x-\sqrt{(1-a x)^{2}-4 b x^{2}}}{2 b x^{2}}=\sum_{k=0}^{\infty} M_{k}(a, b) x^{k}
$$

was discovered. It was pointed out in [2] that

$$
M_{n}(1,1)=M_{n}, \quad M_{n}(2,1)=C_{n+1}, \quad \text { and } \quad M_{n}(3,1)=H_{n},
$$

where $H_{n}$ denote the restricted hexagonal numbers and were described in [17].

For more information on many results, applications, and generalizations of the Motzkin numbers $M_{n}$, please refer to $[1,2,16,18,19]$ and the closely related references therein.

From (1.2) and (1.3), it is easy to see that $m_{n}^{(u, l, d)}=m_{n}^{(d, l, u)}$. Comparing (1.2) with (1.4) reveals that $M_{k}(a, b)$ and $m_{k}^{(u, l, d)}$ are equivalent to each other and satisfy

$$
M_{k}(a, b)=m_{n}^{(1, a, b)}=m_{k}^{(b, a, 1)} \quad \text { and } \quad m_{k}^{(u, l, d)}=M_{k}(l, u d) .
$$

Therefore, it suffices to consider the generalized Motzkin numbers $M_{k}(a, b)$, rather than the $(u, l, d)$-Motzkin numbers $m_{n}^{(u, l, d)}$, in this paper.

The main aim of this paper is to establish explicit formulas for the Motzkin numbers $M_{k}$ and the generalized Motzkin numbers $M_{k}(a, b)$. As consequences, two explicit formulas for the restricted hexagonal numbers $H_{n}$ are derived.

Our main results in this paper can be stated as the following theorems.

Theorem 1 For $k \geq 0$, the Motzkin numbers $M_{k}$ can be computed by

$$
M_{k}=\frac{9}{8}\left(\frac{3}{2}\right)^{k} \sum_{\ell=0}^{k+2}\left(\frac{2}{3}\right)^{\ell} \frac{(2 \ell-3) ! !}{\ell !}\left(\begin{array}{c}
\ell \\
k-\ell+2
\end{array}\right)
$$


where $\left(\begin{array}{l}p \\ q\end{array}\right)=0$ for $q>p \geq 0$ and the double factorial of negative odd integers $-(2 n+1)$ is defined by

$$
[-(2 n+1)] ! !=\frac{(-1)^{n}}{(2 n-1) ! !}=(-1)^{n} \frac{2^{n} n !}{(2 n) !}, \quad n=0,1, \ldots
$$

Theorem 2 For $k \geq 0$ and $a, b \in \mathbb{N}$, the generalized Motzkin numbers $M_{k}(a, b)$ can be computed by

$$
M_{k}(a, b)=\frac{1}{2 b}\left(\frac{4 b-a^{2}}{2 a}\right)^{k+2} \sum_{\ell=0}^{k+2}\left(\frac{2 a^{2}}{4 b-a^{2}}\right)^{\ell} \frac{(2 \ell-3) ! !}{\ell !}\left(\begin{array}{c}
\ell \\
k-\ell+2
\end{array}\right) .
$$

Consequently, the Catalan numbers $C_{k}$ and the restricted hexagonal numbers $H_{k}$ can be computed by

$$
C_{k}=2^{k} \frac{(2 k-1) ! !}{(k+1) !}
$$

and

$$
H_{k}=(-1)^{k} \frac{25}{72}\left(\frac{5}{6}\right)^{k} \sum_{\ell=0}^{k+2}(-1)^{\ell}\left(\frac{18}{5}\right)^{\ell} \frac{(2 \ell-3) ! !}{\ell !}\left(\begin{array}{c}
\ell \\
k-\ell+2
\end{array}\right),
$$

respectively.

Theorem 3 For $n \geq 0$ and $a, b \in \mathbb{N}$, the generalized Motzkin numbers $M_{n}(a, b)$ can be computed by

$$
M_{n}(a, b)= \begin{cases}1, & n=0 \\
\frac{a^{2}}{2 b}\left(\frac{4 b-a^{2}}{2 a}\right)^{n} \sum_{k=0}^{n}\left(\frac{2 a^{2}}{4 b-a^{2}}\right)^{k} \frac{(2 k+1) ! !}{(k+2) !}\left(\begin{array}{l}
k+2 \\
n-k
\end{array}\right), & n \in \mathbb{N} .\end{cases}
$$

Consequently, equation (1.9) for the Catalan numbers $C_{n}$ is valid, the Motzkin numbers $M_{n}$ and the restricted hexagonal numbers $H_{k}$ can be computed by

$$
M_{n}= \begin{cases}1, & n=0, \\
\frac{1}{2}\left(\frac{3}{2}\right)^{n} \sum_{k=0}^{n}\left(\frac{2}{3}\right)^{k} \frac{(2 k+1) ! !}{(k+2) !}\left(\begin{array}{c}
k+2 \\
n-k
\end{array}\right), & n \in \mathbb{N},\end{cases}
$$

and

$$
H_{n}= \begin{cases}1, & n=0, \\
(-1)^{n} \frac{9}{2}\left(\frac{5}{6}\right)^{n} \sum_{k=0}^{n}(-1)^{k}\left(\frac{18}{5}\right)^{k} \frac{(2 k+1) ! !}{(k+2) !}\left(\begin{array}{c}
k+2 \\
n-k
\end{array}\right), & n \in \mathbb{N},\end{cases}
$$

respectively. 


\section{Proofs of main results}

Now we are in a position to prove our main results.

Proof of Theorem 1 From (1.1), it follows that

$$
\sqrt{1-2 x-3 x^{2}}=1-x-2 \sum_{k=0}^{\infty} M_{k} x^{k+2}
$$

This implies that

$$
M_{k}=-\frac{1}{2} \frac{1}{(k+2) !} \lim _{x \rightarrow 0}\left(\sqrt{1-2 x-3 x^{2}}\right)^{(k+2)}, \quad k \geq 0 .
$$

In combinatorial analysis, the Faà di Bruno formula plays an important role and can be described in terms of the Bell polynomials of the second kind

$$
\mathrm{B}_{n, k}\left(x_{1}, x_{2}, \ldots, x_{n-k+1}\right)=\sum_{\substack{1 \leq i \leq n, \ell_{i} \in\{0\} \cup \mathbb{N} \\ \sum_{i=1}^{n} i \ell_{i}=n \\ \sum_{i=1}^{n} \ell_{i}=k}} \frac{n !}{\prod_{i=1}^{n-k+1} \ell_{i} !} \prod_{i=1}^{n-k+1}\left(\frac{x_{i}}{i !}\right)^{\ell_{i}}
$$

for $n \geq k \geq 0$, see [20], p.134, Theorem A, by

$$
\frac{\mathrm{d}^{n}}{\mathrm{~d} t^{n}}[f \circ h(t)]=\sum_{k=0}^{n} f^{(k)}(h(t)) \mathrm{B}_{n, k}\left(h^{\prime}(t), h^{\prime \prime}(t), \ldots, h^{(n-k+1)}(t)\right)
$$

for $n \geq 0$; see [20], p.139, Theorem C. The Bell polynomials of the second kind $\mathrm{B}_{n, k}\left(x_{1}, x_{2}\right.$, $\left.\ldots, x_{n-k+1}\right)$ satisfy the formula

$$
\mathrm{B}_{n, k}\left(a b x_{1}, a b^{2} x_{2}, \ldots, a b^{n-k+1} x_{n-k+1}\right)=a^{k} b^{n} \mathrm{~B}_{n, k}\left(x_{1}, x_{2}, \ldots, x_{n-k+1}\right)
$$

for $n \geq k \geq 0$; see [20], p.135. In [21], Theorem 4.1, [10], Eq. (2.8), and [22], Section 3, it was established that

$$
\mathrm{B}_{n, k}(x, 1,0, \ldots, 0)=\frac{(n-k) !}{2^{n-k}}\left(\begin{array}{l}
n \\
k
\end{array}\right)\left(\begin{array}{c}
k \\
n-k
\end{array}\right) x^{2 k-n}, \quad n \geq k \geq 0 .
$$

Then, for $k \geq 0$, we have

$$
\begin{aligned}
\left(\sqrt{1-2 x-3 x^{2}}\right)^{(k+2)} & =\sum_{\ell=0}^{k+2}\left\langle\frac{1}{2}\right\rangle_{\ell}\left(1-2 x-3 x^{2}\right)^{1 / 2-\ell} \mathrm{B}_{k+2, \ell}(-2-6 x,-6,0, \ldots, 0) \\
& \rightarrow \sum_{\ell=0}^{k+2}\left\langle\frac{1}{2}\right\rangle_{\ell} \mathrm{B}_{k+2, \ell}(-2,-6,0, \ldots, 0) \\
& =\sum_{\ell=0}^{k+2}\left\langle\frac{1}{2}\right\rangle_{\ell}(-6)^{\ell} \mathrm{B}_{k+2, \ell}\left(\frac{1}{3}, 1,0, \ldots, 0\right) \\
& =\sum_{\ell=0}^{k+2}(-1)^{\ell}\left\langle\frac{1}{2}\right\rangle_{\ell} \frac{(k-\ell+2) !}{2^{k-2 \ell+2} 3^{\ell-k-2}}\left(\begin{array}{c}
k+2 \\
\ell
\end{array}\right)\left(\begin{array}{c}
\ell \\
k-\ell+2
\end{array}\right)
\end{aligned}
$$


as $x \rightarrow 0$, where

$$
\langle x\rangle_{n}= \begin{cases}x(x-1) \cdots(x-n+1), & n \geq 1, \\ 1, & n=0,\end{cases}
$$

denotes the falling factorial of $x \in \mathbb{R}$. Consequently, by (2.1), it follows that

$$
M_{k}=-\frac{9}{8}\left(\frac{3}{2}\right)^{k} \frac{1}{(k+2) !} \sum_{\ell=0}^{k+2}(-1)^{\ell}\left\langle\frac{1}{2}\right\rangle_{\ell}\left(\frac{4}{3}\right)^{\ell}(k-\ell+2) !\left(\begin{array}{c}
k+2 \\
\ell
\end{array}\right)\left(\begin{array}{c}
\ell \\
k-\ell+2
\end{array}\right)
$$

for $k \geq 0$, which can be rewritten as (1.7). The proof of Theorem 1 is complete.

Proof of Theorem 2 From (1.4), it is derived that

$$
\sqrt{(1-a x)^{2}-4 b x^{2}}=1-a x-2 b \sum_{k=0}^{\infty} M_{k}(a, b) x^{k+2} .
$$

This implies that

$$
M_{k}(a, b)=-\frac{1}{2 b} \frac{1}{(k+2) !} \lim _{x \rightarrow 0}\left[\sqrt{(1-a x)^{2}-4 b x^{2}}\right]^{(k+2)}, \quad k \geq 0 .
$$

By virtue of (2.2), (2.3), and (2.4), it follows that

$$
\begin{aligned}
{\left[\sqrt{(1-a x)^{2}-4 b x^{2}}\right]^{(k+2)} } \\
=\sum_{\ell=0}^{k+2}\left\langle\frac{1}{2}\right\rangle_{\ell}\left[(1-a x)^{2}-4 b x^{2}\right]^{1 / 2-\ell} \\
\quad \times \mathrm{B}_{k+2, \ell}\left(-2\left[a+\left(4 b-a^{2}\right) x\right], 2\left(a^{2}-4 b\right), 0, \ldots, 0\right) \\
\rightarrow \sum_{\ell=0}^{k+2}\left\langle\frac{1}{2}\right\rangle_{\ell} \mathrm{B}_{k+2, \ell}\left(-2 a, 2\left(a^{2}-4 b\right), 0, \ldots, 0\right) \\
=\sum_{\ell=0}^{k+2}\left\langle\frac{1}{2}\right\rangle_{\ell}\left[2\left(a^{2}-4 b\right)\right]^{\ell} \mathrm{B}_{k+2, \ell}\left(\frac{a}{4 b-a^{2}}, 1,0, \ldots, 0\right) \\
=\sum_{\ell=0}^{k+2}\left\langle\frac{1}{2}\right\rangle_{\ell}\left[2\left(a^{2}-4 b\right)\right]^{\ell} \frac{(k-\ell+2) !}{2^{k-\ell+2}}\left(\begin{array}{c}
k+2 \\
\ell
\end{array}\right)\left(\begin{array}{c}
\ell \\
k-\ell+2
\end{array}\right)\left(\frac{a}{4 b-a^{2}}\right)^{2 \ell-k-2}
\end{aligned}
$$

as $x \rightarrow 0$. Substituting this into (2.5) and simplifying yield

$$
M_{k}(a, b)=-\frac{1}{2 b} \sum_{\ell=0}^{k+2}\left\langle\frac{1}{2}\right\rangle_{\ell}\left[2\left(a^{2}-4 b\right)\right]^{\ell} \frac{1}{2^{k-\ell+2}} \frac{1}{\ell !}\left(\begin{array}{c}
\ell \\
k-\ell+2
\end{array}\right)\left(\frac{a}{4 b-a^{2}}\right)^{2 \ell-k-2}
$$

for $k \geq 0$, which can be further rearranged as (1.8).

Letting $(a, b)=(2,1)$ and $(a, b)=(3,1)$, respectively, in $(1.8)$ and considering the last two relations in (1.5) lead to (1.9) and (1.10) immediately. The proof of Theorem 2 is complete. 
Proof of Theorem 3 For $\left|x\left[\left(a^{2}-4 b\right) x-2 a\right]\right|<1$, the generating function $M_{a, b}(x)$ in (1.4) can be expanded into

$$
\begin{aligned}
M_{a, b}(x) & =\frac{1}{2 b x^{2}}\left[1-a x-\sqrt{1-2 a x+\left(a^{2}-4 b\right) x^{2}}\right] \\
& =\frac{1}{2 b x^{2}}\left\{1-a x-\sum_{k=0}^{\infty}\left\langle\frac{1}{2}\right\rangle_{k} \frac{x^{k}\left[\left(a^{2}-4 b\right) x-2 a\right]^{k}}{k !}\right\} \\
& =\frac{1}{2 b x^{2}}\left\{-\frac{a^{2}-4 b}{2} x^{2}-\sum_{k=2}^{\infty}\left\langle\frac{1}{2}\right\rangle_{k} \frac{x^{k}\left[\left(a^{2}-4 b\right) x-2 a\right]^{k}}{k !}\right\} \\
& =-\frac{1}{2 b}\left\{\frac{a^{2}-4 b}{2}+\sum_{k=2}^{\infty}\left\langle\frac{1}{2}\right\rangle_{k} \frac{x^{k-2}\left[\left(a^{2}-4 b\right) x-2 a\right]^{k}}{k !}\right\} .
\end{aligned}
$$

By (1.4) once again, it follows that

$$
\begin{aligned}
M_{n}(a, b) & =\frac{1}{n !} \lim _{x \rightarrow 0}\left[M_{a, b}(x)\right]^{(n)} \\
& =-\frac{1}{2 b} \frac{1}{n !} \lim _{x \rightarrow 0}\left\{\frac{a^{2}-4 b}{2}+\sum_{k=2}^{\infty}\left\langle\frac{1}{2}\right\rangle_{k} \frac{x^{k-2}\left[\left(a^{2}-4 b\right) x-2 a\right]^{k}}{k !}\right\}^{(n)},
\end{aligned}
$$

which means that

$$
\begin{aligned}
M_{0}(a, b) & =-\frac{1}{2 b} \lim _{x \rightarrow 0}\left\{\frac{a^{2}-4 b}{2}+\sum_{k=2}^{\infty}\left\langle\frac{1}{2}\right\rangle_{k} \frac{x^{k-2}\left[\left(a^{2}-4 b\right) x-2 a\right]^{k}}{k !}\right\} \\
& =-\frac{1}{2 b}\left[\frac{a^{2}-4 b}{2}+\left\langle\frac{1}{2}\right\rangle_{2} \frac{4 a^{2}}{2 !}\right] \\
& =1
\end{aligned}
$$

and

$$
\begin{aligned}
M_{n}(a, b)= & -\frac{1}{2 b} \frac{1}{n !} \lim _{x \rightarrow 0} \sum_{k=2}^{\infty}\left\langle\frac{1}{2}\right\rangle_{k} \frac{\left\{x^{k-2}\left[\left(a^{2}-4 b\right) x-2 a\right]^{k}\right\}^{(n)}}{k !} \\
= & -\frac{1}{2 b} \frac{1}{n !} \lim _{x \rightarrow 0} \sum_{k=0}^{\infty}\left\langle\frac{1}{2}\right\rangle_{k+2} \frac{\left\{x^{k}\left[\left(a^{2}-4 b\right) x-2 a\right]^{k+2}\right\}^{(n)}}{(k+2) !} \\
= & -\frac{1}{2 b} \frac{1}{n !} \lim _{x \rightarrow 0} \sum_{k=0}^{\infty} \frac{\langle 1 / 2\rangle_{k+2}}{(k+2) !} \\
& \times\left\{\sum_{\ell=0}^{k+2}\left(\begin{array}{c}
k+2 \\
\ell
\end{array}\right)\left(a^{2}-4 b\right)^{\ell}(-2 a)^{k-\ell+2} x^{k+\ell}\right\}^{(n)} \\
= & -\frac{1}{2 b} \frac{1}{n !} \lim _{x \rightarrow 0} \sum_{k=0}^{\infty} \frac{\langle 1 / 2\rangle_{k+2}}{(k+2) !} \sum_{\ell=0}^{k+2}\left(\begin{array}{c}
k+2 \\
\ell
\end{array}\right)\left(a^{2}-4 b\right)^{\ell}(-2 a)^{k-\ell+2}\left(x^{k+\ell}\right)^{(n)} \\
= & \frac{1}{2 b} \lim _{x \rightarrow 0} \sum_{k=0}^{\infty} \frac{(2 k+1) ! !}{(k+2) !} \sum_{\ell=n-k}^{k+2}\left(\begin{array}{c}
k+2 \\
\ell
\end{array}\right)\left(\frac{4 b-a^{2}}{2}\right)^{\ell} a^{k-\ell+2}\left(\begin{array}{c}
k+\ell \\
n
\end{array}\right) x^{k+\ell-n}
\end{aligned}
$$




$$
\begin{aligned}
& =\frac{1}{2 b} \sum_{k=0}^{n} \frac{(2 k+1) ! !}{(k+2) !}\left(\begin{array}{c}
k+2 \\
n-k
\end{array}\right)\left(\frac{4 b-a^{2}}{2}\right)^{n-k} a^{2 k-n+2} \\
& =\frac{a^{2}}{2 b}\left(\frac{4 b-a^{2}}{2 a}\right)^{n} \sum_{k=0}^{n} \frac{(2 k+1) ! !}{(k+2) !}\left(\begin{array}{l}
k+2 \\
n-k
\end{array}\right)\left(\frac{2 a^{2}}{4 b-a^{2}}\right)^{k}
\end{aligned}
$$

for $n \in \mathbb{N}$. In conclusion, equation (1.11) follows.

Taking $(a, b)=(2,1),(a, b)=(1,1)$, and $(a, b)=(3,1)$, respectively, in $(1.11)$ and considering the three relations in (1.5) lead to (1.9), (1.12), and (1.13) readily. The proof of Theorem 3 is complete.

\section{Remarks}

Finally, we list several remarks.

Remark 1 The explicit formula (1.8) is a generalization of (1.7).

Remark 2 Equation (1.9) and many other alternative formulas for the Catalan numbers $C_{k}$ can also be found in $[3-6,8,9,12-14]$ and the closely related references therein.

Remark 3 By the second relation in (1.6), equation (1.3) can be reformulated as

$$
M_{n}(a, b)=a^{n} \sum_{j=0}^{n / 2} \frac{1}{j+1}\left(\begin{array}{c}
2 j \\
j
\end{array}\right)\left(\begin{array}{c}
n \\
2 j
\end{array}\right)\left(\frac{b}{a^{2}}\right)^{j}
$$

which is different from the two equations (1.8) and (1.11).

Remark 4 Making use of any one among equations (1.8), (1.11), and (3.1), we can present the first nine generalized Motzkin numbers $M_{n}(a, b)$ for $0 \leq n \leq 8$ and $a, b \in \mathbb{N}$ as follows:

$$
\begin{aligned}
& 1, \quad a, \quad a^{2}+b, \quad a\left(a^{2}+3 b\right), \quad a^{4}+6 a^{2} b+2 b^{2}, \quad a\left(a^{4}+10 a^{2} b+10 b^{2}\right), \\
& a^{6}+15 a^{4} b+30 a^{2} b^{2}+5 b^{3}, \quad a\left(a^{6}+21 a^{4} b+70 a^{2} b^{2}+35 b^{3}\right), \\
& a^{8}+28 a^{6} b+140 a^{4} b^{2}+140 a^{2} b^{3}+14 b^{4} .
\end{aligned}
$$

In particular, the first nine restricted hexagonal numbers $H_{n}$ for $0 \leq n \leq 8$ are

$1, \quad 3, \quad 10, \quad 36, \quad 137, \quad 543, \quad 2,219, \quad 9,285, \quad 39,587$.

\section{Conclusions}

By the Faà di Bruno formula and some properties of the Bell polynomials of the second kind, we establish two explicit formulas for the Motzkin numbers, the generalized Motzkin numbers, and the restricted hexagonal numbers. 


\section{Author details}

'State Key Laboratory of Integrated Service Networks, Xidian University, Xi'an, Shaanxi 710071, China. ${ }^{2}$ Department of Mathematics and Physics, Weinan Normal University, Weinan, Shaanxi 714009, China. ${ }^{3}$ Department of Mathematics, College of Science, Tianjin Polytechnic University, Tianjin, 300387, China. ${ }^{4}$ Institute of Mathematics, Henan Polytechnic University, Jiaozuo, Henan 454010, China.

\section{Acknowledgements}

The first author was partially supported by China Postdoctoral Science Foundation with Grant Number 2015M582619.

Received: 25 November 2016 Accepted: 31 January 2017 Published online: 15 February 2017

\section{References}

1. Donaghey, R, Shapiro, LW: Motzkin numbers. J. Comb. Theory, Ser. A 23, 291-301 (1977)

2. Wang, Y, Zhang, Z-H: Combinatorics of generalized Motzkin numbers. J. Integer Seq. 18(2), 15.2 .4 (2015)

3. Koshy, T: Catalan Numbers with Applications. Oxford University Press, Oxford (2009)

4. Stanley, RP: Catalan Numbers. Cambridge University Press, New York (2015). doi:10.1017/CBO9781139871495

5. Liu, F-F, Shi, X-T, Qi, F: A logarithmically completely monotonic function involving the gamma function and originating from the Catalan numbers and function. Glob. J. Math. Anal. 3, 140-144 (2015). doi:10.14419/gjma.v3i4.5187

6. Mahmoud, M, Qi, F: Three identities of the Catalan-Qi numbers. Mathematics 4(2), 35 (2016). doi:10.3390/math4020035

7. Qi, F, Guo, B-N: Logarithmically complete monotonicity of a function related to the Catalan-Qi function. Acta Univ. Sapientiae Math. 8, 93-102 (2016). doi:10.1515/ausm-2016-0006

8. Qi, F, Guo, B-N: Logarithmically complete monotonicity of Catalan-Qi function related to Catalan numbers. Cogent Math. 3, 1179379 (2016). doi:10.1080/23311835.2016.1179379

9. Qi, F, Mahmoud, M, Shi, X-T, Liu, F-F: Some properties of the Catalan-Qi function related to the Catalan numbers. SpringerPlus 5, 1126 (2016). doi:10.1186/s40064-016-2793-1

10. Qi, F, Shi, X-T, Liu, F-F, Kruchinin, DV: Several formulas for special values of the Bell polynomials of the second kind and applications. J. Appl. Anal. Comput. (2017, in press); (ResearchGate Technical Report (2015). Available online at doi:10.13140/RG.2.1.3230.1927)

11. Qi, F, Shi, X-T, Mahmoud, M, Liu, F-F: Schur-convexity of the Catalan-Qi function related to the Catalan numbers. Tbilisi Math. J. 9(2), 141-150 (2016). Available online at http://dx.doi.org/10.1515/tmj-2016-0026.

12. Qi, F, Shi, X-T, Mahmoud, M, Liu, F-F: The Catalan numbers: a generalization, an exponential representation, and some properties. J. Comput. Anal. Appl. 23, 937-944 (2017)

13. Shi, X-T, Liu, F-F, Qi, F: An integral representation of the Catalan numbers. Glob. J. Math. Anal. 3, 130-133 (2015). doi:10.14419/gjma.v3i3.5055

14. Qi, F: Some properties and generalizations of the Catalan, Fuss, and Fuss-Catalan numbers. ResearchGate Research (2015). Available online at doi:10.13140/RG.2.1.1778.3128

15. Mansour, T, Schork, M, Sun, Y: Motzkin numbers of higher rank: generating function and explicit expression. J. Integer Seq. 10, 07.7.4 (2007)

16. Sun, Z-W: Congruences involving generalized central trinomial coefficients. Sci. China Math. 57, 1375-1400 (2014). doi:10.1007/s11425-014-4809-z

17. Harary, F, Read, RC: The enumeration of tree-like polyhexes. Proc. Edinb. Math. Soc. (2) 17, 1-13 (1970)

18. Lengyel, T: Exact $p$-adic orders for differences of Motzkin numbers. Int. J. Number Theory 10, 653-667 (2014). doi:10.1142/S1793042113501157

19. Lengyel, T: On divisibility properties of some differences of Motzkin numbers. Ann. Math. Inform. 41, 121-136 (2013)

20. Comtet, L: Advanced Combinatorics: The Art of Finite and Infinite Expansions, Revised and Enlarged edn. Reidel, Dordrecht (1974)

21. Guo, B-N, Qi, F: Explicit formulas for special values of the Bell polynomials of the second kind and the Euler numbers. ResearchGate Technical Report (2015). Available online at doi:10.13140/2.1.3794.8808

22. Qi, F, Zheng, M-M: Explicit expressions for a family of the Bell polynomials and applications. Appl. Math. Comput. 258 597-607 (2015). doi:10.1016/j.amc.2015.02.027

\section{Submit your manuscript to a SpringerOpen ${ }^{\ominus}$ journal and benefit from:}

- Convenient online submission

Rigorous peer review

- Immediate publication on acceptance

- Open access: articles freely available online

- High visibility within the field

- Retaining the copyright to your article 